\title{
Identification of risk factors for hyperamylasemia during laparoscopy-assisted surgery for colorectal cancer: a retrospective cohort study
}

Jizhun Zhang ( $\nabla$ zhangjizhun2007@163.com )

Shandong Provincial Hosptial Affiliated to Shandong University https://orcid.org/0000-0001-73839577

\section{Chunjian Wang}

Weifang Traditional Chinese Hosptial

\section{Lipan Peng}

Shandong Provincial Hosptial Affiliated to Shandong University

\section{Tao Xu}

Shandong Provincial Hosptial Affiliated to Shandong University

\section{Jiyang Zuo}

Shandong Provincial Hosptial Affiliated to Shandong University

\section{Hongqing Zhuo}

Shandong Provincial Hosptial Affiliated to Shandong University

\section{Leping Li}

Shandong Provincial Hosptial Affiliated to Shandong University

\section{Fengying Du}

Shandong Provincial Hosptial Affiliated to Shandong University

\section{Research Article}

Keywords: Laparoscopy, Carbon dioxide pneumoperitoneum, Colorectal cancer, Hyperamylasemia, Risk factor

Posted Date: April 27th, 2020

DOl: https://doi.org/10.21203/rs.3.rs-23748/v1

License: (c) (1) This work is licensed under a Creative Commons Attribution 4.0 International License. Read Full License 


\section{Abstract}

Background: Many complications are frequently reported in the carbon dioxide pneumoperitoneum surgery, except hyperamylasemia occurring in pancreas.

Methods: Using data from 380 patients with colorectal cancer registered for a prospective cohort study comparing postoperative serum amylase level, risk factors for postoperative hyperamylasemia and surgical complications were identified by multivariate logistic regression analysis.

Results: Hyperamylasemia occurred in 140 patients, and a total of 52 patients in 380 patients had shortterm postoperative complications, including 25 patients with hyperamylasemia in which 2 patients with acute pancreatitis. The method of operation, injury of pancreas during operation, the duration of pneumoperitoneum and intraoperative blood loss were significant predictors of hyperamylasemia. However, the economic significance of this phenomenon is not certain.

Conclusion: High incidence of hyperamylasemia in laparoscopy-assisted surgery for colorectal cancer, the incidence of postoperative infectious complications will increase significantly. Surgical treatment of rectal cancer, operation around the pancreas, intraoperative blood loss, duration of pneumoperitoneum should be considered when planning surgery. Although patient's hospital cost and postoperative hospital stay were well documented, it is unclear whether this is of any economic significance.

Trial registration: The study was registered with the China Clinical Trial Registry with registration number ChiCTR1800015311 and was registered on March 22, 2018.

\section{Background}

Colorectal cancer is the third most common cancer in the world accounting for above 1.8 million new cases and 881 thousand deaths in 2018(1). In recent decades, laparoscopic surgery has progressively replaced open-colorectal surgery owing to advantageous short-term outcomes, such as less pain, reduced blood loss, and improved recovery time(2-4). And laparoscopic surgery in patients with rectal cancer was associated with rates of locoregional recurrence and disease-free and overall survival similar to those for open surgery(5-7). In China, based on convenient carbon dioxide and wider indications for laparoscopic surgery, laparoscopic surgery with carbon dioxide pneumoperitoneum has been a better choice for patients who suffering colorectal cancer. A large number of studies have found that laparoscopic surgery can make damage to patients' lung(8), heart(9), kidney(10) and liver(11), also result in hypercapnia(12) and immunosuppression(13), while no studies of pancreas have been reported. Hyperamylasemia, referring to abnormal rise of serum amylase, is mostly considered as a manifestation of pancreatic tissue damage. Elevated serum amylase and/or lipase, particularly when over three times more than the normal level, is diagnostic of acute pancreatitis. In response to this phenomenon, we attempted to observe the effect of laparoscopic surgery on pancreas and define risk factors for hyperamylasemia during colorectal cancer surgery and analyze whether hyperamylasemia will affect the postoperative patient's living conditions and economic status or not. 


\section{Methods}

We prospectively included all patients with colorectal cancer from July 2015 to December 2017 at the Shandong Provincial Hospital affiliated to Shandong University, China. The patients who have or had pancreatic, salivary gland-related, biliary-related disease, whose tumor invaded pancreas or who have suffering heavy abdominal surgery were excluded. Besides, patients who have chosen laparoscopic surgery with carbon dioxide pneumoperitoneum and whose pathological result confirmed colorectal cancer were eligible to participate. Institutional review board approval was obtained prior to data abstraction and individual patient consent was also obtained. Martinelli et al. defined the level of serum amylase more than $100 \mathrm{U} / \mathrm{L}$ as hyperamylasemia(14), Christoforidis et al. were more than $180 \mathrm{U} / \mathrm{L}(15)$ and Grimes et al. were more than $276 \mathrm{U} / \mathrm{L}(16)$. Based on the laboratory data from our hospital, the level of serum amylase more than $135 \mathrm{U} / \mathrm{L}$ at surgery 24 hours later was considered as hyperamylasemia in our study, and serum amylase test in the third, fifth and seventh day were performed after operation.

Variables analyzed included patients' physiological characteristics, specifically sex, age, body mass index (BMI), concomitant disease, pathological diagnosis and postoperative complications. A number of kinds of factors associated with laboratory tests and the situation during surgery were also evaluated: serum amylase, urine amylase, hemoglobin, white blood cells, lymphocytes, albumin and prothrombin time; the method of operation, grade of American Society of Anesthesiologists (grade of ASA), intraoperative pancreatic operation, the body position during operation, the method of anesthesia, the pressure of pneumoperitoneum, the duration of pneumoperitoneum, the duration of operation, intraoperative fluid volume, intraoperative urine volume, intraoperative blood loss and intraoperative blood transfusion volume. Besides, postoperative hospital stay and hospital cost of patients were compared to observe if postoperative hyperamylasemia would make an influence in patients' living and economic status.

Factors that might affect the risk of postoperative hyperamylasemia and short-term complications were evaluated by univariate analysis using cross-tabulations and T-tests with appropriate use of Fischer's modification were used for continuous variables, whereas the Wilcoxon rank sum test was used for nonparametric tests. The independent contribution of various factors was assessed by multivariate logistic regression analysis with Forward Stepwise (Likelihood Ratio). And then, nomogram based on the independent risk factors was constructed. Model checking with the Hosmer-Lemeshow test and receiver operating characteristic curve was used to confirm fit of the model. Heteroscedasticity of the residuals and robust standard errors after excluding variables with large deviance residuals was also used to double check the regression model. Two-sided $P$ values are presented and $P$ value below 0.05 was considered significant. Statistical analysis was performed using SPSS 25.0 and R 3.5.1.

\section{Results}

Finally, three hundred and eighty patients were included in this study various in 41 to 77 years old, composed of 248 men and 132 women, and according to the occurrence of hyperamylasemia, they were divided into hyperamylasemia group and normal group. There were 226 patients with pre-operative 
comorbidities in our study, including 28 cases with cardiovascular diseases, 172 cases with metabolic syndromes, 22 cases with pulmonary diseases and 24 cases with anemia. One hundred and twenty-two patients with 1 type of complications and even one hundred and four cases with 2 or more types of comorbidities. Two hundred and eighty-four cases suffered rectal cancer, 88 cases suffered colon cancer, 8 cases suffered rectosigmoid junction cancer, and all patients underwent international standard surgical procedures. Characteristics of the 380 patients are given in Table 1. Unfortunately, the distribution of pneumoperitoneum pressure is severely uneven, and most values of the pneumoperitoneum pressure is $13 \mathrm{mmHg}$, so the pneumoperitoneal pressure is not analyzed.

\section{Hyperamylasemia and its effect on short-term prognosis}

We consulted the records of 380 patients, in which 140 patients (36.8\%) developed hyperamylasemia based on the level of serum amylase after surgery. One hundred and thirty-seven patients returned to normal level of serum amylase on the third day after operation, one case returned to normal on the fifth day and 2 cases returned to normal on the seventh day after operation. Postoperative short-term complications were identified in 52 patients (13.7\%) and 25 cases belong to hyperamylasemia group including 2 cases with acute pancreatitis. In the hyperamylasemia group, 12 patients (8.57\%) had postoperative infectious complications, while the normal group had only 9 patients (3.75\%). There was a statistically significant difference in the incidence of postoperative infectious complications between two groups $(P$ value $=0.047)$, supported by Table 2 .

\section{Univariate analysis of factors}

The results of univariate analyses with 23 risk factors for hyperamylasemia are summarized in Table 3. Only the method of operation ( $P$ value $<0.001)$, injury of pancreas during operation $(P$ value $=0.047)$, the body position during operation ( $P$ value $=0.003)$, the duration of operation $(P$ value $<0.001)$, the duration of pneumoperitoneum ( $P$ value $<0.001)$, intraoperative fluid volume $(P$ value $=0.030)$ and intraoperative blood loss $(P$ value $<0.001)$ were identified as significant independent risk factors for postoperative hyperamylasemia. In the method of operation, patients with laparoscopic radical resection for rectal cancer, known as Miles Operation, are more prone to hyperamylasemia.

\section{Logistic regression analysis defines risk factors}

Whether hyperamylasemia or not as the dependent variable and seven factors underwent univariate analysis as the covariate, multivariate logistic regression analysis identified the method of operation, injury of pancreas during operation, intraoperative blood loss and the duration of pneumoperitoneum as significant independent risk factors for postoperative hyperamylasemia (Table 4), which is consistent with our clinical experience.

\section{The model establishment and drawing of the nomogram}

The model was established based on the four independent risk factors and presented on the nomogram (supported in Figure 1). We used the Receiver Operating Characteristic (ROC) curve to test the validity of 
this model. Fortunately, the Area Under Curve (AUC) of this model is 0.774, which shown in Figure 2.

\section{Comparison of postoperative hospital stay and hospital cost between two groups}

The hospital cost and postoperative hospital stay of hyperamylasemia group and normal group were counted separately, and it wasn't found that the postoperative hospital stay of patients from hyperamylasemia group was longer than that of patients from normal group significantly (8.76 d \pm $5.751: 7.81 \mathrm{~d} \pm 2.716, P$ value $=0.066$ ), which is close to significance. Unfortunately, there was also no statistical difference in hospital cost between two groups (53180.0 yuan \pm 15669.9 : 51646.5 yuan \pm $12819.3, P$ value $=0.301)$, but the average hospital cost of patients from hyperamylasemia group was slightly higher than that from normal group (Table 5). Although these changes in postoperative hospital stay and hospital cost are well documented, it is unclear whether this is of any economic significance. This is likely to be associated with transient hyperamylasemia that does not show significant signs and symptoms, so it does not attract the attention of medical staff.

\section{Discussion}

As far as we know, this is the first study designed to examine risk factors for hyperamylasemia during the laparoscopic surgery with carbon dioxide pneumoperitoneum for colorectal cancer at a single facility, which involves more than 300 patients. Our team recorded and analyzed not only reported possible risk factors (the method of operation(17), the method of anesthesia(18), etc.) but also several original potential factors. It is reported that the incidence of hyperamylasemia in abdominal surgery with open operation ranges from $9 \%$ to $25 \%(18-22)$, especially $19 \%(17)$ in colonic surgery, while the incidence of hyperamylasemia in laparoscopic Roux-en-Y gastric bypass surgery is $71 \%(23)$. However, we couldn't find related reports from open operation in the past two decades, or any more reports from laparoscopic surgery. In our study, the incidence of hyperamylasemia was relatively high (36.84\%, 140 of 380 patients), postoperative serum amylase increased mostly transiently, 97.86\% (137 of 140 patients) of patients returned to normal condition within 3 days and $0.71 \%$ ( 1 of 140 patients) of patients recovered within 5 days, others recovered within 7 days. The results of transiently elevated amylase are similar to the report from Morrissey' team (18). Two patients with hyperamylasemia progressed to acute pancreatitis and cured under appropriate and timely treatment and 138 patients showed asymptomatic hyperamylasemia. We analyzed that it may be related to the self-repair of pancreas. Takaori et al.(24) think that the initial stage of pancreatic tissue's damage is a reversible process and a lesser degree of inflammation can be restored by its own compensatory function, acute pancreatitis develops only when necrotic lesions that are severe enough to irreversibly affect pancreas growth and development. We guess that it is also the reason why hyperamylasemia does not necessarily accompany acute pancreatitis. As for hyperamylasemia, on the one hand, it may be due to damage to the pancreatic tissue during surgery, and on the other hand, it probably because the application of carbon dioxide pneumoperitoneum affects the microcirculation of the pancreas. 
The results of our study show that the method of operation is an independent risk factor for postoperative hyperamylasemia. The mechanism may be during the operation of laparoscopic radical resection for rectal cancer, also known as Miles Operation, doctors need to remove a wide range of tissues and need to make sputum at the colon, which causes unintentional excessive pulling of the pancreas or accidental damage to the pancreas. Serum amylase increases after abdominal surgery is mainly caused by pancreatic tissue injury, especially the upper position of surgery, in which may directly damage pancreas, the incidence of hyperamylasemia is significantly increased(25). Baniel et al. also think direct pancreatic trauma with retractors may be blamed(26). To confirm this view, we reviewed recent reports of laparoscopic cholecystectomy, in which is much closer to pancreas. It does have a high incidence of hyperamylasemia during laparoscopic cholecystectomy ranging from $10 \%$ to $45 \%(27-30)$. Compared with our study, the incidence is obviously lower in the reports from Yong Fan et al. $(10 \%, 6$ of 60 patients)(30) and Ming Guo Tian et al.(12\%, 4 of 33 patients)(29). However, in these two studies, the number of total cases was only 33 or 60 , such a small sample size may be the key to the low incidence of hyperamylasemia.

$\mathrm{CO}_{2}$ pneumoperitoneum during laparoscopic surgery may have a significant effect on organs, especially intra-abdominal organ function, which may be the main influencing factor of hyperamylasemia in laparoscopic surgery(8-13). The results of this study show that longer duration of pneumoperitoneum can significantly increase the incidence of postoperative hyperamylasemia. Too high intra-abdominal pressure, caused by $\mathrm{CO}_{2}$ pneumoperitoneum, can oppress intraperitoneal blood vessels, especially the veins, to reduce blood perfusion of abdominal organs, while pancreatic and other substantive organs more easily affected $(31,32)$. The pancreas has a unique microcirculation structure, pancreatic lobules supplied by the independent lobular artery and lack of collateral circulation, which resulting in lower compensatory capacity for ischemia and prone to ischemia and ischemia-reperfusion injury $(24,33)$. This is an important factor in pancreatic injury. Takaori et al. analyzed that high intra-abdominal pressure may press the bile duct and the main pancreatic duct, causing the deposition of pancreatic juice and even countercurrent, which result in the damage with pancreatic tissue(34). Thus, we suspect that the incidence of hyperamylasemia in laparoscopic surgery (37\%) is higher than that in open surgery (9 to $25 \%(18-22)$ ) due to the large pressure from pneumoperitoneum. Unfortunately, in this study, we recorded the pressure of pneumoperitoneal in all cases, but the data were severely biased and unevenly distributed, leading us to not further explore the relationship between the pressure of pneumoperitoneum and hyperamylasemia. It is reasonable to speculate that information bias or too few cases cause such a result. It may also be explained that just when a certain pressure of pneumoperitoneum reaches a suitable time, the degree of pancreatic injury would vary with pressure. As for intraoperative blood loss, which is an important cause for hyperamylasemia, this is easier to explain. The more blood loss, the smaller blood supply of pancreatic tissue and the more likely to result in ischemic injury.

We additionally investigated some biochemical indicators that could reflect the condition of the patients' body, such as hemoglobin, albumin, etc., but did not find a link between them and hyperamylasemia. It is very likely that the degree of change of these indicators before and after surgery is related to 
hyperamylasemia, and we didn't record the values of these indicators after surgery. We plan to focus on this in future studies. Similarly, our result is consistent with the result of Morrissey et al.(18) that there is no correlation between the method of anesthesia and hyperamylasemia. In contrast, previous reports mentioned no adverse effects on the outcome of patients with hyperamylasemia(17), our conclusion also verified this view in short-term postoperative complications $\left(\chi^{2}=14.298\right.$, $P$ value $=0.112$, while patients in hyperamylasemia group are more susceptible to infectious complications $\left(\chi^{2}=3.937, P\right.$ value $=0.047$, $O R=2.283$ ). We suspect that the sample size of our study is still insufficient, which also has to attract the attention of all medical workers.

By comparing the hospital cost and postoperative hospital stay of patients from two groups, it is easy to find that hyperamylasemia could not cause the patients' postoperative hospital stay to prolong (Hyperamylasemia group: Normal group $=8.76 \mathrm{~d} \pm 5.751$ :7.81 $\mathrm{d} \pm 2.716, P$ value $=0.066$ ), which might be related to the patient with asymptomatic hyperamylasemia needing a certain amount of time to complete the self-repair of pancreas, but this has not exceeded the sustainable level of self-repairing in patient's pancreas. Specifically, if reach a level higher than pancreatic self-repairing ability, the postoperative hospital stay would be expected to prolong. As for the hospital cost, there was no statistically significant difference (Hyperamylasemia group: Normal group $=53180.0$ yuan \pm 15669.9 : 51646.5 yuan \pm 12819.3 , $P$ value $=0.301$ ). Our team speculated that this is most likely because asymptomatic hyperamylasemia did not attract enough attention of medical staff resulting that they did nothing for these patients. Although the data demonstrate that there is no difference in hospital cost, patients could not benefit from it, after all, a transient increase in serum amylase might impose a certain burden on pancreas. When medical staff pays enough attention to asymptomatic hyperamylasemia, how the patient's hospital cost change, our team plans to follow up on this aspect. From the perspective of health economics and patient's satisfaction, doctors and nurses should pay attention to asymptomatic hyperamylasemia.

Not only that, but we also plan to design a series of hyperamylasemia diagnostic management system, including improve the nomogram scoring system that can be used to determine hyperamylasemia in early postoperative period, and postoperative management procedures for patients with hyperamylasemia to improve their living conditions and rationally optimize the allocation of medical resources.

\section{Conclusions}

The incidence of hyperamylasemia in laparoscopic colorectal surgery is higher than that of open surgery, and the risk of postoperative infection will be significantly increased. Although patient's hospital cost and postoperative hospital stay were well documented, it is unclear whether this is of any economic significance. For patients with hyperamylasemia, the postoperative hospital stay and hospital cost were not significantly changed than these of normal group. The method of operation, injury of pancreas during operation, longer duration of pneumoperitoneum and heavy intraoperative blood loss are independent risk factors for hyperamylasemia after laparoscopic surgery. When there is an operation near the 
pancreas during surgery, it is essential for the surgeon to repeatedly demonstrate the surgical plan before surgery and carry out operative manipulations carefully in view of the risk of hyperamylasemia occurs.

\section{Abbreviations}

\begin{tabular}{ll} 
Abbreviations & Full name \\
\hline BMI & body mass index \\
\hline grade of ASA & grade of American Society of Anesthesiologists \\
\hline the Receiver Operating Characteristic curve & ROC curve \\
\hline Area Under Curve & AUC
\end{tabular}

\section{Declarations}

\section{Ethics approval and consent to participate}

Institutional review board approval was obtained prior to data abstraction by Ethics Committee of Shandong Provincial Hospital Affiliated to Shandong University (NO.2017544) and individual patient consent was also obtained.

\section{Consent for publication}

Consent for publication was obtained in this study.

\section{Availability of data and material}

The data that support the findings of this study are available from the China Clinical Trial Registry but restrictions apply to the availability of these data, which were used under license for the current study, and so are not publicly available. Data are however available from the authors upon reasonable request and with permission of the China Clinical Trial Registry.

\section{Competing interests}

The authors declare that they have no competing interests.

\section{Funding}

This work was supported by the National Natural Science Foundation of China (Grant No.81702363) in study design, collection, analysis, interpretation of data and writing of the report.

\section{Authors' contributions}


Jizhun Zhang and Leping Li made contributions to conception and design. Jiyang Zuo and Tao Xu collected patient data. Chunjian Wang and Lipan Peng analyzed and interpreted the patient data regarding hyperamylasemia. Fengying Du and Hongqing Zhuo were major contributors in writing the manuscript. All authors read and approved the final manuscript.

\section{Acknowledgements}

This work was supported by the National Natural Science Foundation of China (Grant No.81702363).

\section{References}

1. Bray F, Ferlay J, Soerjomataram I, Siegel RL, Torre LA, Jemal A. Global cancer statistics 2018 : GLOBOCAN estimates of incidence and mortality worldwide for 36 cancers in 185 countries. CA: a cancer journal for clinicians. 2018;68(6):394-424.

2. Veldkamp R, Kuhry E, Hop WC, Jeekel J, Kazemier G, Bonjer HJ, et al. Laparoscopic surgery versus open surgery for colon cancer: short-term outcomes of a randomised trial. Lancet Oncology. 2005;6(7):477-84.

3. Lujan J, Valero G, Biondo S, Espin E, Parrilla P, Ortiz H. Laparoscopic versus open surgery for rectal cancer: results of a prospective multicentre analysis of 4,970 patients. Surgical Endoscopy. 2013;27(1):295.

4. Mh VDP, Haglind E, Cuesta MA, Fürst A, Lacy AM, Hop WC, et al. Laparoscopic versus open surgery for rectal cancer (COLOR II): short-term outcomes of a randomised, phase 3 trial. Lancet Oncology. 2013;14(3):210-8.

5. Denz C, Siegel L, Lehmann KJ, Dagorn JC, Fiedler F. Is hyperlipasemia in critically ill patients of clinical importance? An observational CT study. Intensive Care Medicine. 2007;33(9):1633-6.

6. Hardt PD, Mayer K, Ewald N. Exocrine pancreatic involvement in critically ill patients. Current Opinion in Clinical Nutrition \& Metabolic Care. 2009;12(2):168-74.

7. Bonjer HJ, Deijen CL, Abis GA, Cuesta MA, van der Pas MH, de Lange-de Klerk ES, et al. A randomized trial of laparoscopic versus open surgery for rectal cancer. N Engl J Med. 2015;372(14):1324-32.

8. Mazzeo, Bordes, Goutorbe, Cungi, P.J, Antonini. Intraperitoneal pneumoperitoneum effects on lung function compared with extraperitoneal pneumoperitoneum effects in anesthetized adults: 5AP2-9. European Journal of Anaesthesiology. 2014;31:81.

9. Hazebroek EJ, Haitsma JJ, Lachmann B, Steyerberg EW, Bruin RWF, Bouvy ND, et al. Impact of carbon dioxide and helium insufflation on cardiorespiratory function during prolonged pneumoperitoneum in an experimental rat model. Surgical Endoscopy. 2002;16(7):1073-8.

10. Demyttenaere S, Feldman LS, Fried GM. Effect of pneumoperitoneum on renal perfusion and function: A systematic review. Surgical Endoscopy. 2007;21(2):152-60.

11. Szold A, Weinbroum AA. Carbon dioxide pneumoperitoneum-related liver injury is pressure dependent: A study in an isolated-perfused organ model. Surgical Endoscopy. 2008;22(2):365-71. 
12. Yavuz $Y$, Rønning $K$, Bakkelund $K$, Lyng $O$, Aadahl $P$, Mårvik R, et al. Hemodynamic and tissue blood flow responses to long-term pneumoperitoneum and hypercapnia in the pig. Surgical Endoscopy \& Other Interventional Techniques. 2006;20(9):1394-401.

13. Hajri A, Mutter D, Wack S, Bastien C, Gury J, Marescaux J, et al. Dual effect of laparoscopy on cellmediated immunity. European surgical research. 2000;32(5):261-6.

14. Martinelli M, Strisciuglio C, Illiceto MT, Cardile S, Guariso G, Vignola S, et al. Natural history of pancreatic involvement in paediatric inflammatory bowel disease. Digestive \& Liver Disease Official Journal of the Italian Society of Gastroenterology \& the Italian Association for the Study of the Liver. 2015;47(5):384.

15. Christoforidis E, Goulimaris I, Kanellos I, Tsalis K, Demetriades C, Betsis D. Post-ERCP pancreatitis and hyperamylasemia: patient-related and operative risk factors. Endoscopy. 2002;34(4):286.

16. Grimes KL, Maciel VH, Mata W, Arevalo G, Singh K, Arregui ME. Complications of laparoscopic transgastric ERCP in patients with Roux-en-Y gastric bypass. Surgical Endoscopy. 2015;29(7):1753-9.

17. Griffith, Leong, Ho, Nyam, Seow-Choen. The significance of hyperamylasaemia after colonic resection. Colorectal Disease the Official Journal of the Association of Coloproctology of Great Britain \& Ireland. 1999;1(6):347-50.

18. Morrissey R, Berk JE, Fridhandler L, Pelot D. The nature and significance of hyperamylasemia follwing operation. Annals of Surgery. 1974;180(1):67.

19. Mahaffey JH, Howard JM. The incidence of postoperative pancreatitis; study of one hundred thirtyone surgical patients, utilizing the serum amylase concentration. Amaarchives of Surgery. 1955;70(3):348.

20. Singh LM, Okukubo F, Jr JP, Salmon J, Howard JM. FURTHER STUDIES ON POSTOPERATIVE PANCREATITIS. Archives of Surgery. 1965;90(1):43.

21. Keighley MR, Johnson AG, Stevens AE. Raised serum amylase after upper abdominal operation. British Journal of Surgery. 1969;56(6):424.

22. Harada K, Kitamura M, Ikenaga T. Isoenzyme study on postoperative transient hyperamylasemia. American Journal of Gastroenterology. 1974;61(3):121.

23. Spector D, Perry Z, Shah S, Kim JJ, Tarnoff ME, Shikora SA. Roux-en-Y gastric bypass: hyperamylasemia is associated with small bowel obstruction. Surgery for Obesity \& Related Diseases. 2015;11(1):38-43.

24. Kadirov RK, Arkhipova SS, Shahmardanova SA, Rizvanov AA. Structural Changes in the Pancreas and Its Blood Vessels at the Early Stages of Ischemia. Bionanoscience. 2016;6(4):1-4.

25. Knight AH, Williams DN, Ellis G, Goldberg DM. Significance of Hyperamylasaemia and Abdominal Pain in Diabetic Ketoacidosis. British Medical Journal. 1973;3(5872):128.

26. Baniel J, Leibovitch I, Foster RS, Rowland RG, Bihrle R, Donohue JP. Hyperamylasemia after postchemotherapy retroperitoneal lymph node dissection for testis cancer. Journal of Urology. 1995;154(4):1373-5. 
27. Isla AM, Griniatsos J, Wan A. A technique for safe placement of a biliary endoprosthesis after laparoscopic choledochotomy. Journal of Laparoendoscopic \& Advanced Surgical Techniques Part A. 2002;12(3):207.

28. Chikamori F, Kuniyoshi N, Kawashima T, Takase Y. Technique of laparoscopic cholecystectomy combined with percutaneous papillary balloon dilatation under general anesthesia for cholecystocholedocholithiasis. Digestive Endoscopy. 2003;15(2):142-6.

29. Tian MG, Shi WJ, Zhong CJ, Zhang XW, Chen TM. Laparoscopic treatment of choledocholithiasis with novel self-releasing biliary stent. Journal of Laparoendoscopic \& Advanced Surgical Techniques Part A. 2009;19(3):405.

30. Fan Y, Liu YY, Wang P, Wang C, Li XS, Kang YX, et al. Study on the efficacies of splenic pedicle transection by using manual manipulation and Endo-GIA procedure for laparoscopic splenectomy. International Journal of Clinical \& Experimental Medicine. 2015;8(10):19430.

31. Gutt CN, Oniu T, Mehrabi A, Schemmer P, Kashfi A, Kraus T, et al. Circulatory and respiratory complications of carbon dioxide insufflation. Dig Surg. 2004;21(2):95-105.

32. Ypsilantis P, Lambropoulou M, Tentes I, Chryssidou M, Georgantas T, Simopoulos C. Room air versus carbon dioxide pneumoperitoneum: effects on oxidative state, apoptosis and histology of splanchnic organs. Surg Endosc. 2016;30(4):1388-95.

33. Bockman DE. Microvasculature of the pancreas. International Journal of Pancreatology. 1992;12(1):11-21.

34. Takaori K, Inoue K, Kogire M, Higashide S, Tun T, Aung T, et al. Effects of endothelin on microcirculation of the pancreas. Life Sciences. 1992;51(8):615-22.

\section{Tables}

Table 1 Characteristics of the 380 patients 
Normal group Hyperamylasemia group

\begin{tabular}{lll}
\hline Age (years) & $60.10 \pm 9.881$ & $61.60 \pm 10.151$ \\
\hline Sex $(\mathrm{M} / \mathrm{F})$ & $152: 88$ & $96: 44$ \\
\hline $\mathrm{BMI}\left(\mathrm{kg} / \mathrm{m}^{2}\right)$ & $24.43 \pm 3.370$ & $24.66 \pm 3.146$ \\
\hline the site of cancer & & \\
\hline Rectal cancer & $164(68.33 \%)$ & $120(85.71 \%)$ \\
\hline Colon cancer & $68(28.33 \%)$ & $20(14.29 \%)$ \\
\hline Rectosigmoid junction cancer & $8(3.33 \%)$ & 0 \\
\hline Concomitant disease & & \\
\hline Cardiovascular disease & $12(5.00 \%)$ & $16(11.43 \%)$ \\
\hline Metabolic syndrome & $95(39.58 \%)$ & $77(55.00 \%)$ \\
\hline Pulmonary disease & $14(5.83 \%)$ & $8(5.71 \%)$ \\
\hline Anemia & $14(5.83 \%)$ & $10(7.14 \%)$ \\
\hline $\mathbf{1}$ type of complication & $34(14.17 \%)$ & $68(48.57 \%)$ \\
\hline $\mathbf{2}$ or more types of complications & $62(25.83 \%)$ & $42(30.00 \%)$ \\
\hline
\end{tabular}

Table 2 Complications in two groups 
Normal group Hyperamylasemia group $P$

\begin{tabular}{llll}
\hline Inflammatory complications & $12(5.00 \%)$ & $8(5.71 \%)$ & 0.764 \\
\hline Ostomy dermatitis & $12(5.00 \%)$ & $6(4.29 \%)$ & \\
\hline Pancreatitis & 0 & $2(1.43 \%)$ & 0.047 \\
\hline Infectious complications & $9(3.75 \%)$ & $12(8.57 \%)$ & \\
\hline Incision infection & $6(2.50 \%)$ & $6(4.29 \%)$ & 0.436 \\
\hline Abdominal infection & 0 & $4(2.86 \%)$ & \\
\hline Respiratory tract infection & $3(1.25 \%)$ & $2(1.43 \%)$ & \\
\hline Fistula & $4(1.67 \%)$ & $4(2.86 \%)$ & 0.899 \\
\hline Rectovaginal fistula & 0 & $1(0.71 \%)$ & \\
\hline Anastomotic fistula & $4(1.67 \%)$ & $3(2.14 \%)$ & $1(0.71 \%)$ \\
\hline Bleeding & $2(0.83 \%)$ & 0 & 0.112 \\
\hline Stress ulcer bleeding & $1(0.42 \%)$ & $1(0.71 \%)$ & \\
\hline Postoperative abdominal bleeding & $1(0.42 \%)$ & $25(17.86 \%)$ & \\
\hline Total & $27(11.25 \%)$ & & \\
\hline
\end{tabular}

Table 3 Univariate analysis of factors in two groups 


\section{Normal group}

the method of operation

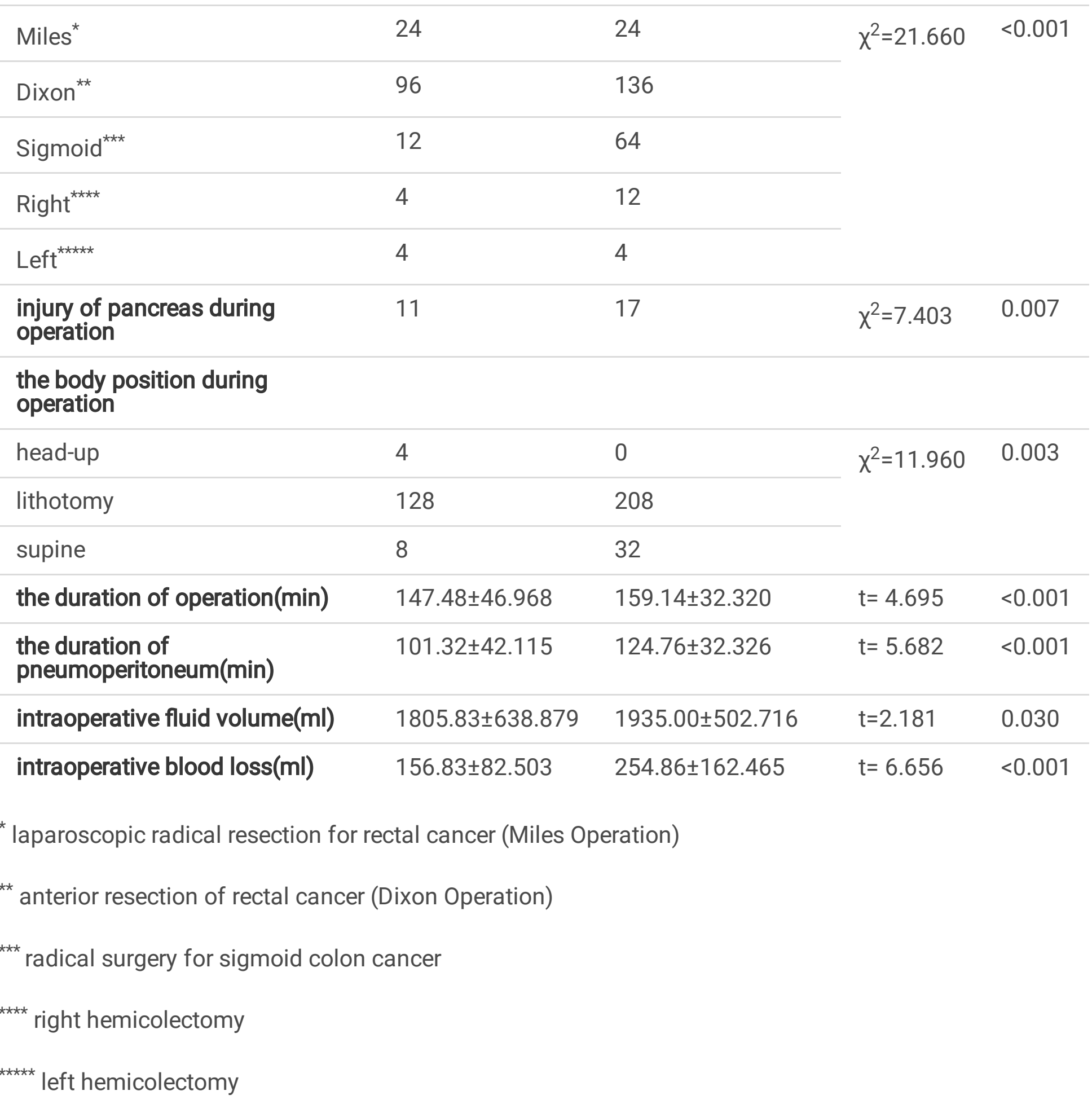

Table 4 the result of Logistic multivariate regression analysis 


\begin{tabular}{|c|c|c|c|c|c|c|c|}
\hline Factors & B & S.E & $\begin{array}{l}\text { Wald } \\
x^{2}\end{array}$ & $\operatorname{Exp}(B)$ & $\begin{array}{l}95 \% \mathrm{Cl} \\
\text { down }\end{array}$ & $\begin{array}{l}95 \% \mathrm{Cl} \\
\text { up }\end{array}$ & $P$ \\
\hline the method of operation & -0.512 & 0.195 & 6.873 & 0.599 & 0.408 & 0.879 & 0.009 \\
\hline $\begin{array}{l}\text { injury of pancreas during } \\
\text { operation }\end{array}$ & 2.271 & 0.533 & 18.141 & 9.686 & 3.407 & 27.540 & $<0.001$ \\
\hline $\begin{array}{l}\text { the duration of } \\
\text { pneumoperitoneum }\end{array}$ & 0.008 & 0.003 & 5.597 & 1.008 & 1.001 & 1.015 & 0.018 \\
\hline intraoperative blood loss & 0.006 & 0.001 & 27.623 & 1.006 & 1.004 & 1.009 & $<0.001$ \\
\hline
\end{tabular}

Table 5 Comparison of postoperative hospital stay and hospital cost between two groups

\begin{tabular}{llll} 
& Normal group & Hyperamylasemia group & $P$ \\
\hline Postoperative hospital stay (days) & & & 0.066 \\
\hline Average & 7.81 & 8.76 & \\
\hline Median & 7 & 7 & 0.301 \\
\hline Standard deviation & 2.716 & 5.751 & \\
\hline Hospital cost (yuan) & & & \\
\hline Average & 51970.53 & 53180.0 & \\
\hline Median & 50785.39 & 51430.17 & \\
\hline Standard deviation & 15274.561 & 15669.9 &
\end{tabular}

\section{Figures}


Points

the method of operation

Injury of pancreas during operation

the duration of pneumoperitoneum (min)

Intraoperative blood loss $(\mathrm{mL})$

Total Points

Risk of Hyperamylasemia
0

10

20

\begin{abstract}
30
\end{abstract}
40

50

60

70

80

90 100

\begin{tabular}{|c|c|c|}
\hline \multirow[b]{2}{*}{ Left } & \multicolumn{2}{|c|}{ Dixon } \\
\hline & Sigmoid & Miles \\
\hline & & Yes \\
\hline
\end{tabular}

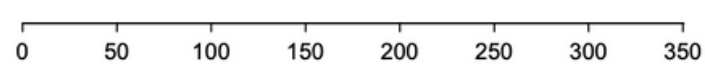

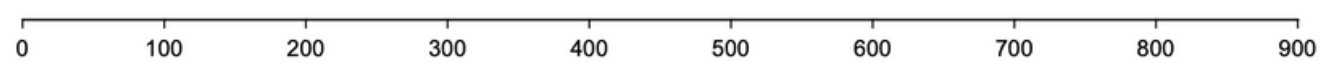

$\begin{array}{lllllll}1 & 60 & 80 & 100 & 120 & 140 & 160\end{array}$

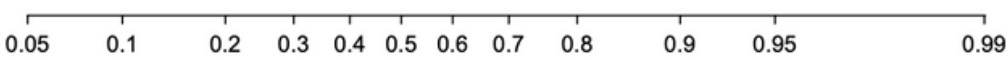

\section{Figure 1}

The nomogram model predicting the risk of postoperative hyperamylasemia. 
ROC Curve

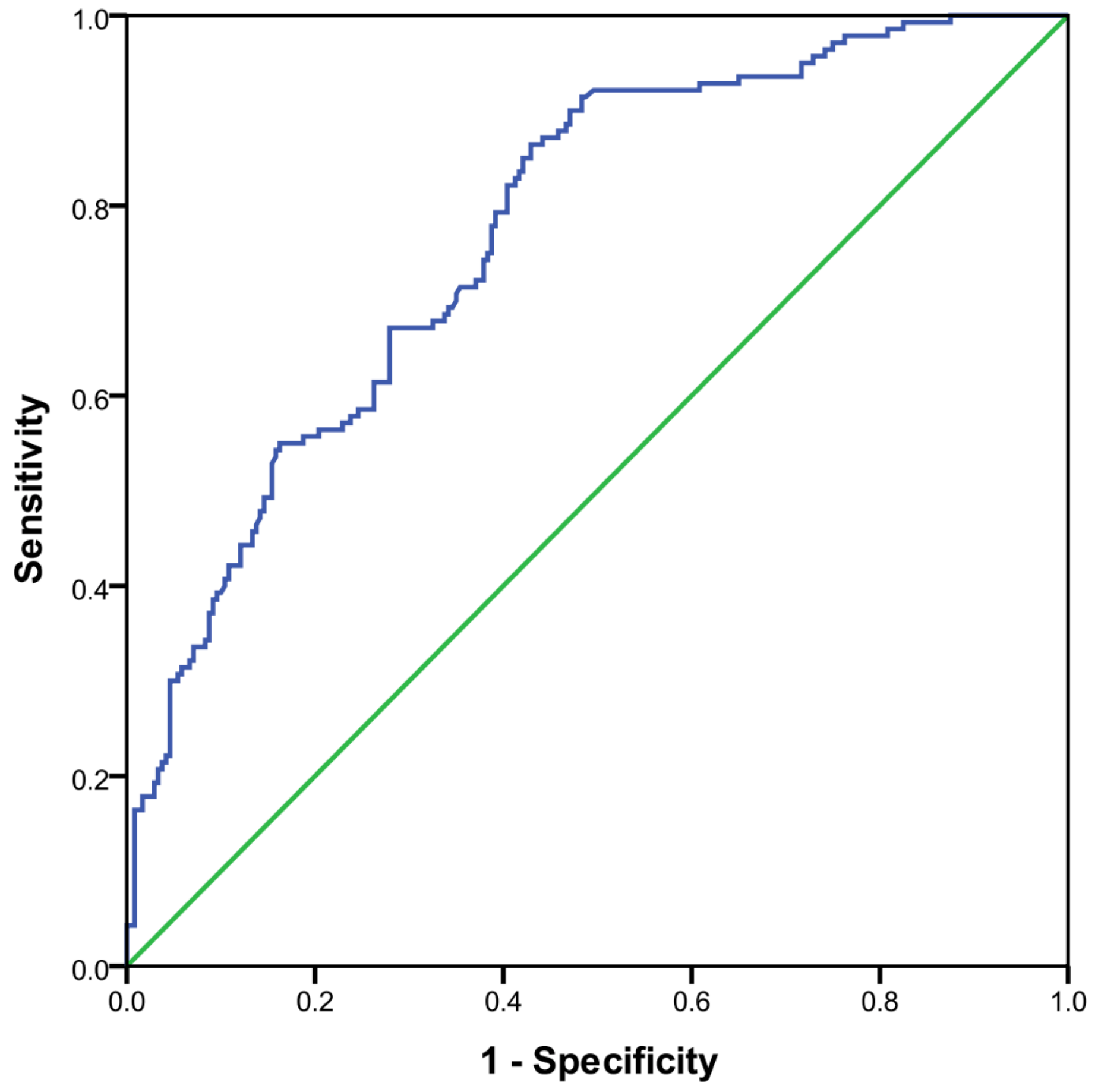

Figure 2

The ROC curve of the nomogram model. 